\title{
Distress and psychosocial risk in families with newly diagnosed pediatric brain tumors
}

\author{
Brandon G. Rocque, MD, MS, ${ }^{1}$ Alexandra Cutillo, MA, ${ }^{2}$ Kathrin Zimmerman, BA, ${ }^{1}$ \\ Anastasia Arynchyna, MPH, ${ }^{1}$ Susan Davies, $\mathrm{PhD},{ }^{3}$ Wendy Landier, $\mathrm{PhD},{ }^{4}$ and \\ Avi Madan-Swain, $\mathrm{PhD}^{4}$
}

\begin{abstract}
'Division of Pediatrics, Department of Neurosurgery, University of Alabama at Birmingham; '2Department of Psychology, University of Alabama at Birmingham; ${ }^{3}$ Department of Health Behavior, School of Public Health, University of Alabama at Birmingham; and ${ }^{4}$ Division of Hematology/Oncology, Department of Pediatrics, University of Alabama at Birmingham, Alabama
\end{abstract}

OBJECTIVE Hospitalization for a newly diagnosed pediatric brain tumor is an extremely stressful time for a family, but this period has not been the focus of rigorous study. The purpose of this study was to quantify distress and psychosocial risk in this population to improve psychosocial care delivery.

METHODS The authors administered the National Comprehensive Cancer Network Distress Thermometer (DT) and the Psychosocial Assessment Tool 2.0 (PAT) to primary caregivers of all children admitted to Children's of Alabama with a new brain tumor between April 2016 and August 2017. The DT is a single-item measure of distress (scale range 0-10). The PAT (range 0-7) stratifies families by risk level: a score less than 1.0 indicates universal risk level (risk typically experienced during hospitalization); a score of 1.0-2.0 indicates targeted risk (specific psychosocial difficulties that impact medical treatment); and a score higher than 2.0 indicates clinically significant risk. Demographic and clinical information was abstracted from each child's medical record. A correlation matrix using nonparametric statistics was created between abstracted data and the DT and PAT scores.

RESULTS Forty primary caregivers were enrolled (of 49 eligible), with the patient age ranging from newborn to 17 years (mean 7.7 years). Twenty-five (63\%) of the children were male, and 24 (60\%) were white, non-Hispanic. Mean and median DT scores were 7.2 (SD 2.6) and 7, respectively. However, 12 (30\%) rated their distress 10/10, and 85\% rated distress 5 or greater. PAT scores ranged from 0.0 to 2.36 with mean and median scores of 0.89 (SD 0.50 ) and 0.86 , respectively. PAT results for $16(40 \%)$ families were in the targeted or clinical range, indicating psychosocial factors that have the potential to interfere with medical treatment. No clinical or demographic variable correlated significantly with the DT or PAT score.

CONCLUSIONS Families of children with newly diagnosed brain tumors experience high levels of distress and psychosocial risk. This work will serve as the foundation for efforts to standardize psychosocial evaluation for newly diagnosed pediatric neurosurgical patients, and to create protocols that organize existing hospital-based psychosocial support services. These efforts have the potential to improve patient and family satisfaction as well as treatment outcomes.

https://thejns.org/doi/abs/10.3171/2018.7.PEDS18297

KEYWORDS distress; psychosocial risk; pediatric brain tumor treatment; caregiver; family; oncology

$\mathrm{T}$ HE diagnosis of a brain tumor in a child is a stressful event for the entire family. The initial hospitalization for surgical treatment carries a myriad of potentially distressing factors for both the patient and family members. Specifically, prior studies have indicated a link between parental stress/distress and anxiety about their child's illness and its impact on child adjustment and coping, potentially leading to maladaptive responses that can affect the entire course of treatment. . $^{3,67,7,10,15}$ Distress is broadly viewed as an emotional response consisting of a range of emotions, including sadness, worry, and fear. ${ }^{5}$ Therefore, a strategy to measure and mitigate family distress has potential benefits to the family not only during the initial hospitalization but also throughout the brain

ABBREVIATIONS DT = Distress Thermometer; PAT = Psychosocial Assessment Tool 2.0 .

SUBMITTED May 17, 2018. ACCEPTED July 26, 2018.

INCLUDE WHEN CITING Published online October 12, 2018; DOI: 10.3171/2018.7.PEDS18297. 
tumor treatment trajectory. Given the dearth of literature documenting family distress in this population, the primary focus of the present study is understanding and delineating family distress.

Psychosocial risk is defined as the balance of factors that contribute to distress, anxiety, and maladaptive coping responses against the resources and resiliency factors available to a family. Studies of pediatric cancer have shown that patients and parents have significant risk of distress at the time of diagnosis., ${ }^{3,7}$ A study in pediatric cancer patients (mostly leukemia patients within 6 weeks of diagnosis) showed that an intervention as simple as providing a summary of psychosocial risk to medical providers can lead to significantly reduced distress 6 months later. ${ }^{2}$ Other research has shown that routine use of psychosocial distress screening measures leads to greater utilization of psychosocial support services. ${ }^{6}$ However, little is documented in the literature regarding the assessment of risk and resiliency factors in families whose child is hospitalized urgently with a brain tumor.

Pediatric patients with brain tumors are a unique population due to the potential for neurological deficits, the near-universal necessity of surgery as a first step in treatment, and the initial uncertainty about whether the tumor is benign or malignant. In an effort to understand these aspects of pediatric brain tumor treatment, we have undertaken a detailed analysis of this population using both quantitative and qualitative research methodologies. The purpose of the present study is to quantify distress among caregivers of children with newly diagnosed brain tumors and to measure psychosocial risk, helping to understand factors that contribute to distress.

\section{Methods}

All English- and Spanish-speaking families of children admitted to Children's of Alabama with a newly diagnosed brain tumor between April 2016 and August 2017 were eligible for inclusion and prospective enrollment. Families were approached by a research assistant who described the purpose of the study and obtained consent from participating parents. Study instruments were administered using a tablet-based platform, with data immediately uploaded into a REDCap research database. When possible, study instruments were administered within 72 hours of diagnosis and before neurosurgical intervention. Families received standard clinical care during the course of the study. Assessment and follow-up by social work, pastoral care, and child life specialists were performed on an asneeded basis, when consulted by the medical team.

\section{Study Instruments}

\section{The Distress Thermometer}

The National Comprehensive Cancer Network Distress Thermometer (DT) is a single-item screening tool designed to assess the level of distress in cancer patients. It is an 11-point visual analog scale that measures the level of distress from 0 to $10 .{ }^{11}$ The DT is fast and easy to administer, and it has been validated for use with pediatric patients and families of children with cancer (including leukemia, musculoskeletal, and other solid tumors), as well as spe- cifically validated for children with brain tumors. ${ }^{4,14}$ Furthermore, the DT has been validated for use immediately after diagnosis as well as at multiple time points during pediatric cancer treatment. ${ }^{13}$ We used the DT to quantify the level of distress in families of children with newly diagnosed brain tumors.

\section{The Psychosocial Assessment Tool}

The Psychosocial Assessment Tool 2.0 (PAT) is a brief, parent-reported measure of psychosocial risk that is typically completed in 5-10 minutes. The PAT is based on the Pediatric Psychosocial Preventative Health Model and provides a stratified assessment of family risk and resilience. ${ }^{8}$ Families receive a total PAT score that allows stratification into one of the following 3 risk levels: universal (minimal risk factors and many resources that assist the family in coping and adjusting to their child's diagnosis and treatment), targeted (families that are at risk in specific domains, have moderate resources to assist them in coping, and will benefit from interventions targeted at identified risk domains), or clinical (many risk factors and limited resources). This instrument has strong content validity and has been found to be both sensitive and specific in detecting clinically significant problems in a sample including children with leukemia, brain tumors, and other solid tumor diagnoses..$^{12}$ In previous studies, the PAT has been used shortly after diagnosis and has been predictive of later psychosocial difficulties. ${ }^{1,6}$

The PAT has 7 empirically derived and clinically meaningful subscales (structure/resource issues, family problems, social support, stress reactions, family beliefs, child problems, and sibling problems) and strong psychometric properties (Cronbach's alpha $=0.81$ for the total score and $0.62-0.81$ for the subscales). The current version of the PAT is written at a 4th grade reading level.

Additional information collected on each patient/family includes age, sex, and race/ethnicity of the patient; private or public insurance; severity of neurological symptoms on presentation (Table 1); tumor location (supratentorial or infratentorial); and whether the histological diagnosis was benign or malignant (Table 2). In general, tumors considered benign were treated with resection alone without additional chemotherapy or radiation therapy. Finally, we also collected information about which psychosocial service providers consulted on each family during the hospitalization.

\section{Statistical Analysis}

The primary goal of this study was to quantify emotional distress and psychosocial risk. Therefore, we used descriptive statistics to report the results from the DT and PAT. To explore for association between clinical and demographic variables and DT scores, we used univariate linear regression analysis, estimating the mean difference in distress level between groups of dichotomous variables and constructing individual $95 \%$ confidence intervals for each variable. Mean difference near zero indicates weak association of that variable with distress level. Univariate linear regression was also used to assess for a relationship between clinical and demographic variables and the PAT score. We then constructed a multivariate linear regression model of DT score, using age, sex, race/ethnicity, 
TABLE 1. Grading of neurological deficits

\begin{tabular}{cl}
\hline Grade & \multicolumn{1}{c}{ Description of Signs or Symptoms } \\
\hline 1 & No deficits or seizures \\
\hline 2 & Mild deficits: cranial nerve palsy, controlled seizures \\
\hline 3 & Moderate deficits: mild hemiparesis, poorly controlled seizures \\
\hline 4 & $\begin{array}{c}\text { Severe deficits: moderate to severe hemiparesis, major stroke, } \\
\text { significant abulia, lethargy, etc. }\end{array}$ \\
\hline
\end{tabular}

type of insurance, single parent, and tumor histology as the independent variables. These variables were selected for inclusion in the multivariate analysis a priori (before beginning data collection). Finally, we used linear regression to assess for association between PAT scores, both overall score as well as subscale scores, and DT score. Significance was set as $\mathrm{p}<0.05$.

\section{Results}

A total of 40 patients were included in the analysis. The study sample included 25 male children (62.5\%) and 15 female children (37.5\%). Twenty-four (60\%) patients were white, non-Hispanic. Patient age at entry into the study ranged from newborn to 17.6 years (mean $8.0 \pm 5.8$ years, median 8.5 years).

Of the 40 patients with a newly diagnosed brain tumor, 15 (38\%) eventually received a diagnosis of a benign tumor. These tumors have WHO grade I classification and are treated with surgery alone, without the addition of chemotherapy or radiation therapy. The remaining 25 (62\%) patients had other brain tumor diagnoses. Ten patients $(25 \%)$ came from families experiencing financial problems, and 14 (35\%) were from single-parent households. Twenty-two (55\%) patients had hydrocephalus on admission. All patients survived hospitalization; 2 (5\%) died over the course of this 1-year study, and 2 (5\%) died after the study was completed. In terms of hospital services provided during the inpatient stay, the number of patients provided specific support services were the following: 2 (5\%) counseling/psychology, 8 (20\%) pastoral care, $22(55 \%)$ social work, and $32(80 \%)$ child life. Fifteen patients received inpatient evaluation and treatment from physical medicine and rehabilitation, and 26 (65\%) were seen in consultation by an oncologist.

The mean DT score was 7.18 (SD 2.59). Likewise, the median DT score was 7 (range 1-10). However, the mode or most common response was 10/10 distress. Univariate analysis determined that there was a significant association between DT and tumor histology, with benign or low-grade tumors associated with lower DT scores (linear regression coefficient $-1.99,95 \% \mathrm{CI}-3.60$ to $-0.38 ; \mathrm{p}=$ $0.017)$. There were no significant associations between DT scores and any other clinical or demographic variable (Table 3). Additionally, there were no significant associations between DT scores and either overall or subscale PAT scores (Table 4).

Table 5 summarizes results from multivariable linear regression. The association between tumor histology and DT did not maintain statistical significance on multivari-
TABLE 2. Classification of tumor histology for included tumors

\begin{tabular}{cl}
\hline \multicolumn{1}{c}{ Benign Tumors } & \multicolumn{1}{c}{ Malignant Tumors } \\
\hline $\begin{array}{l}\text { Choroid plexus papilloma } \\
\text { Tectal glioma }\end{array}$ & Pilomyxoid astrocytoma \\
$\begin{array}{c}\text { Ganglioglioma (WHO } \\
\text { grade I) }\end{array}$ & Grade IV astrocytoma (GBM) \\
Cesmoplastic infantile & Diffuse intrinsic pontine glioma \\
ganglioglioma & Germinoma \\
Polymorphous low-grade & Ependymoma (WHO grade II) \\
neuroepithelial tumor & Anaplastic ependymoma (WHO grade III) \\
of the young & Craniopharyngioma \\
Pilocytic astrocytoma & Embryonal tumor, NOS (WHO grade IV) \\
& Disseminated glioneuronal tumor \\
& Anaplastic astrocytoma (WHO grade III) \\
& Gliomatosis cerebri \\
& Immature teratoma \\
& Anaplastic ganglioglioma \\
& Optic glioma \\
& Infantile fibrosarcoma \\
\hline
\end{tabular}

NOS $=$ not otherwise specified.

ate analysis (linear regression coefficient $-1.89,95 \%$ CI -3.86 to $0.71 ; p=0.058)$. None of the other associations were statistically significant: DT scores versus age $(p=$ $0.47)$, sex $(\mathrm{p}=0.33)$, race/ethnicity $(\mathrm{p}=0.47)$, insurance $(\mathrm{p}$ $=0.53)$, or single-parent household $(\mathrm{p}=0.69)$.

PAT overall scores ranged from 0 to 2.36 (mean $0.89 \pm$ 0.50 , median 0.86). Of the 3 PAT risk levels, 24 families $(60 \%)$ scored in the universal range (i.e., minimal risk factors and many resources that help them cope successfully and adapt to their child's diagnosis); 15 families (37.5\%) scored in the targeted range (i.e., families that have some identified areas of risk or moderate resources and are likely to benefit from targeted interventions to reduce symptoms), and 1 family (2.5\%) scored in the clinical range (i.e., many risk factors with few resources, high risk for interference with clinical care). Table 6 summarizes the associations between PAT scores and clinical/demographic data. In the univariate linear regression analysis, benign tumors were associated with higher mean PAT overall score (linear regression coefficient $0.321,95 \%$ CI 0.005 to $0.64 ; \mathrm{p}=0.047$ ). There was also an association between the overall PAT score and consultation with child life services, with families who did receive child life services having a significantly lower overall PAT score than those who did not (regression coefficient $-0.478,95 \%$ CI -0.85 to $0.11 ; p=0.013)$. In regard to other hospital services offered during inpatient hospital stay, there were no significant associations between PAT scores and services provided by counseling/psychology $(\mathrm{p}=0.12)$, pastoral care $(p=0.11)$, social work $(p=0.37)$, rehabilitation medicine $(\mathrm{p}=0.76)$, and oncology $(\mathrm{p}=0.34)$.

\section{Discussion}

The purpose of this study was to quantify distress and measure psychosocial risk in caregivers of children with a newly diagnosed brain tumor. There are several important findings in this study. First, as hypothesized, levels 
TABLE 3. DT relationship with clinical and demographic variables (univariate linear regression)

\begin{tabular}{|c|c|c|c|c|c|}
\hline Distress Thermometer vs ... & Value & Mean DT Score & Linear Regression Coefficient & $95 \% \mathrm{Cl}$ & $p$ Value \\
\hline Mean age at diagnosis, yrs & 8.0 & 7.18 & -0.070 & -0.215 to 0.075 & 0.34 \\
\hline Sex & & & 0.147 & -1.59 to 1.88 & 0.87 \\
\hline Male & 25 & 7.12 & & & \\
\hline Female & 15 & 7.27 & & & \\
\hline Race/ethnicity & & & 0.140 & -1.61 to 1.89 & 0.87 \\
\hline White, non-Hispanic & 24 & 7.13 & & & \\
\hline Other & 15 & 7.27 & & & \\
\hline Insurance & & & 0.785 & -0.833 to 2.40 & 0.33 \\
\hline Public & 23 & 7.22 & & & \\
\hline Private & 14 & 6.71 & & & \\
\hline Single parent & & & -0.93 & -2.66 to 0.81 & 0.29 \\
\hline Yes & 14 & 6.57 & & & \\
\hline No & 26 & 7.50 & & & \\
\hline Family money problems & & & 0.30 & -1.64 to 2.24 & 0.76 \\
\hline Yes & 10 & 7.40 & & & \\
\hline No & 30 & 7.10 & & & \\
\hline Neurological deficit grade at admission & & & $N A^{*}$ & $N A^{*}$ & $0.84^{*}$ \\
\hline 1 & 20 & 7.05 & & & \\
\hline 2 & 9 & 7.44 & & & \\
\hline 3 & 9 & 7.11 & & & \\
\hline 4 & 2 & 7.50 & & & \\
\hline Hydrocephalus present at admission & & & -1.0 & -2.65 to 0.066 & 0.23 \\
\hline Yes & 22 & 6.73 & & & \\
\hline No & 18 & 7.72 & & & \\
\hline Tumor histology & & & -1.99 & -3.60 to -0.38 & 0.017 \\
\hline Benign & 15 & 5.93 & & & \\
\hline Malignant & 25 & 7.92 & & & \\
\hline Counseling/psychology & & & -0.18 & -4.04 to 3.67 & 0.92 \\
\hline Yes & 2 & 7.0 & & & \\
\hline No & 38 & 7.18 & & & \\
\hline Pastoral care & & & 0.25 & -1.85 to 2.35 & 0.81 \\
\hline Yes & 8 & 7.38 & & & \\
\hline No & 32 & 7.13 & & & \\
\hline Social work & & & 0.419 & -1.26 to 2.10 & 0.62 \\
\hline Yes & 22 & 7.36 & & & \\
\hline No & 18 & 6.94 & & & \\
\hline Child life & & & -0.719 & -2.81 to 1.37 & 0.49 \\
\hline Yes & 32 & 7.03 & & & \\
\hline No & 8 & 7.75 & & & \\
\hline Rehabilitation medicine & & & 0.893 & -0.82 to 2.60 & 0.30 \\
\hline Yes & 15 & 7.73 & & & \\
\hline No & 25 & 6.84 & & & \\
\hline Oncology & & & 0.379 & -1.38 to 2.14 & 0.67 \\
\hline Yes & 26 & 7.31 & & & \\
\hline No & 14 & 6.93 & & & \\
\hline Mean admission to survey administration, days $†$ & 2.85 & & 0.093 & -0.243 to 0.428 & 0.58 \\
\hline Survey administered before surgery† & & & 0.233 & -1.45 to 1.91 & 0.78 \\
\hline Yes & 21 & 7.29 & & & \\
\hline No & 19 & 7.05 & & & \\
\hline
\end{tabular}

$\mathrm{NA}=$ not applicable.

Boldface type indicates statistical significance.

* Linear regression cannot be used to analyze the relationship between an independent categorical variable with more than 2 possible values and a continuous outcome variable. However, the $p$ value derived from 1-way ANOVA is reported here and shows that the relationship between neurological deficits on admission and DT score is not significant.

† Post hoc analysis. 
TABLE 4. DT relationship with PAT overall and component scores

\begin{tabular}{|c|c|c|c|}
\hline \multirow[b]{2}{*}{ PAT Score } & \multicolumn{3}{|c|}{ Relationship w/ DT Score (linear regression) } \\
\hline & Linear Regression Coefficient & $95 \% \mathrm{Cl}$ & $\mathrm{p}$ Value \\
\hline $\begin{array}{l}\text { Overall score } \\
\text { Mean } 0.89 \\
\text { Range } 0-2.36 \\
\text { SD } 0.5\end{array}$ & -0.223 & -1.93 to 1.48 & 0.79 \\
\hline $\begin{array}{l}\text { Family structure \& resources score } \\
\text { Mean } 0.15 \\
\text { Range } 0-0.86 \\
\text { SD } 0.18\end{array}$ & -0.61 & -5.34 to 4.12 & 0.80 \\
\hline $\begin{array}{l}\text { Social support score } \\
\text { Mean } 0.08 \\
\text { Range } 0-0.75 \\
\text { SD } 0.19\end{array}$ & -3.56 & -7.86 to 0.74 & 0.10 \\
\hline $\begin{array}{l}\text { Patient problems score (patient }<2 \text { yrs) } \\
\text { Mean } 0.14 \\
\text { Range } 0-0.5 \\
\text { SD } 0.18\end{array}$ & 4.67 & -3.66 to 12.99 & 0.25 \\
\hline $\begin{array}{l}\text { Patient problems score (patient age } \geq 2 \text { yrs) } \\
\text { Mean } 0.15 \\
\text { Range } 0-1.0 \\
\text { SD } 0.21\end{array}$ & -0.59 & -5.28 to 4.10 & 0.80 \\
\hline $\begin{array}{l}\text { Sibling problems score }(\geq 2) \\
\text { Mean } 0.11 \\
\text { Range } 0-0.35 \\
\text { SD } 0.13\end{array}$ & -6.47 & -15.34 to 2.41 & 0.14 \\
\hline $\begin{array}{l}\text { Sibling problems score (both }<2 \& \geq 2 \text { yrs) } \\
\text { Mean } 0.16 \\
\text { Range } 0-0.43 \\
\text { SD } 0.24\end{array}$ & -6.49 & -119.60 to 106.61 & 0.60 \\
\hline $\begin{array}{l}\text { Caregiver problems score } \\
\text { Mean } 0.13 \\
\text { Range } 0-0.4 \\
\text { SD } 0.13\end{array}$ & 2.35 & -4.32 to 9.00 & 0.71 \\
\hline $\begin{array}{l}\text { Caregiver stress reactions score } \\
\text { Mean } 0.18 \\
\text { Range } 0-0.8 \\
\text { SD } 0.17\end{array}$ & 1.39 & -3.58 to 6.35 & 0.58 \\
\hline $\begin{array}{l}\text { Family beliefs score } \\
\text { Mean } 0.12 \\
\text { Range } 0-0.5 \\
\text { SD } 0.14\end{array}$ & 3.89 & -1.98 to 9.76 & 0.19 \\
\hline
\end{tabular}

of distress in this population are very high. While the DT scores ranged from 1 to 10 (of possible scores $0-10$ ), the single most common response was $10 / 10$ distress, with a mean and median around 7/10. Clearly, parents of children with newly diagnosed brain tumors are highly distressed. However, although we hypothesized that demographic variables such as single-parent household or public insurance would correlate with DT scores, this was not the case. This is important since it indicates that, regardless of demographic variables, all families with a child newly diagnosed with a brain tumor are at risk for distress.

Interestingly, families of children with low-grade or benign tumors showed lower levels of distress. We admin- istered the DT before surgery in most cases, but it was always given several days before the histological diagnosis was available. Therefore, families did not know whether the tumor was benign or malignant when they completed the surveys. It is possible that children with malignant tumors had more severe or troubling symptoms that led to more parental distress. Our analysis included an assessment of the level of neurological symptoms at admission, and this was not found to be statistically significant. However, the method used for assessment of neurological symptoms may not have been sensitive in differentiating subtle signs or symptoms that a parent might notice, in turn leading to higher levels of distress. It is also possible 
TABLE 5. Multivariate linear regression analysis of DT versus key clinical and demographic variables (defined a priori)

\begin{tabular}{lccc}
\hline $\begin{array}{c}\text { DT Multivariate } \\
\text { Linear Regression }\end{array}$ & Coefficient & $95 \% \mathrm{Cl}$ & $\begin{array}{c}\mathrm{p} \\
\text { Value }\end{array}$ \\
\hline Age & -0.058 & -0.22 to 0.10 & 0.47 \\
\hline Sex & 0.927 & -0.96 to 2.82 & 0.33 \\
\hline Race/ethnicity & 0.639 & -1.16 to 2.44 & 0.47 \\
\hline Insurance & 0.547 & -1.20 to 2.30 & 0.53 \\
\hline Tumor histology & -1.89 & -3.86 to 0.71 & 0.058 \\
\hline Single parent & -0.379 & -2.32 to 1.57 & 0.69 \\
\hline
\end{tabular}

that the treating team conveyed an impression of the seriousness of the tumor to parents even before establishment of a formal diagnosis. This transfer of information, which we have no way to quantify in this study, could also influence the level of distress. This result did not maintain statistical significance in the multivariate model.

This study used the PAT to assess psychosocial risk. The level of risk observed in this study is consistent with that in previous studies of pediatric cancer., ${ }^{1,6,12}$ Here again, we observed a significant association between tumor histology and PAT score. However, in this case, benign tumors were associated with higher PAT scores, indicating a higher level of psychosocial risk. The PAT measures family social factors and, as discussed above, was administered before diagnosis was known. Therefore, we would not expect a relationship between tumor diagnosis and PAT score. Because we tested 12 variables for association with the overall PAT score, a correction for multiple measures is appropriate. Using Bonferroni correction, this association did not maintain statistical significance.

We observed a significant association between PAT score and services provided by child life specialists; families receiving a higher overall PAT score were less likely to receive services from a child life specialist. This finding suggests that families at higher risk who may have benefited from evaluation or treatment by child life services did not receive them. The reason for this is unclear but may in part stem from the lack of an established protocol for psychosocial support. Specifically, the PAT was administered for research purposes and was not used to guide the use of psychosocial support services. Child life specialists play a crucial role in providing education to children and teens about their medical diagnosis, treatment, and side effects and assist them in preparing for medical procedures. They help normalize the hospital experience and enhance coping through play and self-expression activities during the medical journey. There is variability in the hospital as to whether the child life service is consulted by the admitting service, or if the service is routinely involved in the assessment of all patients. (At the time of this study, all oncology and neurooncology patients as well as nearly all patients in the Pediatric ICU were evaluated by the child life specialist.)

With all other hospital services, we observed no significant association between the hospital support services that a family receives and their DT or PAT scores. We believe that this negative finding is an important observation. The lack of association between the DT and PAT scores and support services may indicate that families in the greatest need of support are not receiving it. This represents a potential gap in care and an opportunity for improvement.

There is a large body of literature documenting family risk and resiliency factors in childhood cancer. In contrast, there is a dearth of literature examining psychosocial risk in families of children with brain tumors. This study is the first to examine distress and psychosocial risk in families with a child newly diagnosed with a brain tumor. Certainly, brain tumor patients are a somewhat unique population, in that they may have neurological deficits, either from the tumor or as a result of surgery, and nearly all children with brain tumors will undergo surgery. The ultimate treatment, including chemotherapy and radiation therapy, depends on tumor histology, molecular diagnostics, and many other factors. The initial assessment and treatment is identical, whether the tumor is high or low grade. Therefore, the period between diagnosis of a tumor and receiving the final histological diagnosis is one of great uncertainty.

The present study identifies an area of need. Based on these findings, our center has begun to implement a protocol for psychosocial support in this population. When a child with a newly diagnosed brain tumor is admitted to the hospital, an assessment that includes the DT and PAT is performed by a lay patient navigator. The DT and PAT are then reviewed by the navigator and shared with the family. Areas of concern can be addressed, either directly by the navigator, or by social work, psychology, or pastoral care as appropriate. The individual items included in the PAT, as well as the subscale summary scores, facilitate the provision of these services, tailored to family need. This model has the potential to help families identify obstacles and find needed support immediately after diagnosis. Further investigation into the efficacy of this program is ongoing.

\section{Limitations}

This study is limited by a relatively small sample size of 40 families and by heterogeneity in patient age, race, and family makeup. However, this sample represents nearly all newly diagnosed brain tumors at our tertiary care center over an 18-month period. As such, it is a good representation of our patient population. Nevertheless, these factors may limit the generalizability of the findings to other centers. The degree of neurological impairment of the child at diagnosis may be a significant factor in family distress. Our method for quantifying neurological impairment is broad by necessity, since there are a large variety of possible symptoms and severity that result from brain tumors. However, this limits the degree to which we can assess the impact of neurological deficit.

\section{Conclusions}

This study quantifies distress and psychosocial risk in a sample of caregivers of children with newly diagnosed brain tumors. These families exhibited high levels of distress, regardless of family structure, economics, child age, tumor type, and other factors. Our findings argue for universal attention to family distress when treating these children. Data from this study will form the basis of an inpatient psychosocial support protocol that is currently 
TABLE 6. Summary of PAT Scores and relationship with study variables

\begin{tabular}{|c|c|c|c|c|}
\hline PAT Overall Score vs... & Mean Overall PAT Score & Coefficient & $95 \% \mathrm{Cl}$ & p Value \\
\hline Age at diagnosis, yrs & 0.89 & -0.01 & -0.038 to 0.018 & 0.49 \\
\hline Sex & & 0.223 & -0.10 to 0.55 & 0.17 \\
\hline Male & 0.81 & & & \\
\hline Female & 1.03 & & & \\
\hline Race/ethnicity & & 0.51 & -0.29 to 0.39 & 0.31 \\
\hline White, non-Hispanic & 0.87 & & & \\
\hline Other & 0.92 & & & \\
\hline Insurance & & -0.275 & -0.58 to 0.26 & 0.072 \\
\hline Public & 0.74 & & & \\
\hline Private & 1.12 & & & \\
\hline Neurological deficits at admission & & $N A^{*}$ & $N A^{*}$ & $0.50^{*}$ \\
\hline 1 & 0.91 & & & \\
\hline 2 & 0.97 & & & \\
\hline 3 & 0.84 & & & \\
\hline 4 & 0.58 & & & \\
\hline Hydrocephalus present on admission & & -0.007 & -0.33 to 0.31 & 0.97 \\
\hline Yes & 0.89 & & & \\
\hline No & 0.89 & & & \\
\hline Tumor histology & & 0.321 & 0.005 to 0.64 & 0.047 \\
\hline Benign & 1.09 & & & \\
\hline Malignant & 0.77 & & & \\
\hline Counseling/psychology & & -0.563 & -1.28 to 0.16 & 0.12 \\
\hline Yes & 0.36 & & & \\
\hline No & 0.92 & & & \\
\hline Pastoral care & & -0.318 & -0.71 to 0.072 & 0.11 \\
\hline Yes & 0.64 & & & \\
\hline No & 0.95 & & & \\
\hline Social work & & -0.143 & -0.46 to 0.18 & 0.37 \\
\hline Yes & 0.83 & & & \\
\hline No & 0.97 & & & \\
\hline Child life & & -0.478 & -0.85 to -0.11 & 0.013 \\
\hline Yes & 0.79 & & & \\
\hline No & 1.27 & & & \\
\hline Rehab & & -0.052 & -0.39 to 0.28 & 0.76 \\
\hline Yes & 0.86 & & & \\
\hline No & 0.91 & & & \\
\hline Oncology & & -0.161 & -0.50 to 0.17 & 0.34 \\
\hline Yes & 0.83 & & & \\
\hline No & 0.99 & & & \\
\hline
\end{tabular}

under design at our institution. We hope that these efforts will contribute to improved future outcomes for this highrisk group of patients and families.

\section{Acknowledgments}

This work was supported by NIH grant no. 1KL2TR001419.

\section{References}

1. Alderfer MA, Mougianis I, Barakat LP, Beele D, DiTaranto
S, Hwang WT, et al: Family psychosocial risk, distress, and service utilization in pediatric cancer: predictive validity of the Psychosocial Assessment Tool. Cancer 115 (18 Suppl):4339-4349, 2009

2. Barrera M, Hancock K, Rokeach A, Atenafu E, Cataudella D, Punnett A, et al: Does the use of the revised Psychosocial Assessment Tool (PATrev) result in improved quality of life and reduced psychosocial risk in Canadian families with a child newly diagnosed with cancer? Psychooncology 23:165-172, 2014

3. Dolgin MJ, Phipps S, Fairclough DL, Sahler OJZ, Askins M, 
Noll RB, et al: Trajectories of adjustment in mothers of children with newly diagnosed cancer: a natural history investigation. J Pediatr Psychol 32:771-782, 2007

4. Goebel S, Mehdorn HM: Measurement of psychological distress in patients with intracranial tumours: the NCCN distress thermometer. J Neurooncol 104:357-364, 2011

5. Holland JC, Bultz BD: The NCCN guideline for distress management: a case for making distress the sixth vital sign. J Natl Compr Canc Netw 5:3-7, 2007

6. Kazak AE, Barakat LP, Hwang WT, Ditaranto S, Biros D, Beele $\mathrm{D}$, et al: Association of psychosocial risk screening in pediatric cancer with psychosocial services provided. Psychooncology 20:715-723, 2011

7. Kazak AE, Brier M, Alderfer MA, Reilly A, Fooks Parker S, Rogerwick $S$, et al: Screening for psychosocial risk in pediatric cancer. Pediatr Blood Cancer 59:822-827, 2012

8. Kazak AE, Rourke MT, Alderfer MA, Pai A, Reilly AF, Meadows AT: Evidence-based assessment, intervention and psychosocial care in pediatric oncology: a blueprint for comprehensive services across treatment. J Pediatr Psychol 32:1099-1110, 2007

9. Lewandowski LA, Baranoski MV: Psychological aspects of acute trauma: intervening with children and families in the inpatient setting. Child Adolesc Psychiatr Clin N Am 3:513-529, 1994

10. Melnyk BM, Alpert-Gillis L, Feinstein NF, Crean HF, Johnson J, Fairbanks E, et al: Creating opportunities for parent empowerment: program effects on the mental health/coping outcomes of critically ill young children and their mothers. Pediatrics 113:e597-e607, 2004

11. National Comprehensive Cancer Network: Distress management. Clinical practice guidelines. J Natl Compr Canc Netw 1:344-374, 2003

12. Pai ALH, Patiño-Fernández AM, McSherry M, Beele D, Alderfer MA, Reilly AT, et al: The Psychosocial Assessment Tool (PAT2.0): psychometric properties of a screener for psychosocial distress in families of children newly diagnosed with cancer. J Pediatr Psychol 33:50-62, 2008

13. Patel SK, Fernandez N, Wong AL, Mullins W, Turk A, Dekel $\mathrm{N}$, et al: Changes in self-reported distress in end-of-life pediatric cancer patients and their parents using the pediatric distress thermometer. Psychooncology 23:592-596, 2014
14. Patel SK, Mullins W, Turk A, Dekel N, Kinjo C, Sato JK: Distress screening, rater agreement, and services in pediatric oncology. Psychooncology 20:1324-1333, 2011

15. Selove R, Kroll T, Coppes M, Cheng Y: Psychosocial services in the first 30 days after diagnosis: results of a web-based survey of Children's Oncology Group (COG) member institutions. Pediatr Blood Cancer 58:435-440, 2012

\section{Disclosures}

The authors report no conflict of interest concerning the materials or methods used in this study or the findings specified in this paper.

\section{Author Contributions}

Conception and design: Rocque, Davies, Landier, Madan-Swain. Acquisition of data: Rocque, Cutillo, Arynchyna. Analysis and interpretation of data: Rocque, Cutillo, Arynchyna, Davies, Landier. Drafting the article: Rocque, Cutillo, Zimmerman, Arynchyna. Critically revising the article: Rocque, Cutillo, Zimmerman, Davies, Landier, Madan-Swain. Reviewed submitted version of manuscript: all authors. Approved the final version of the manuscript on behalf of all authors: Rocque. Statistical analysis: Rocque. Administrative/technical/material support: Rocque, Arynchyna. Study supervision: Davies, Landier, Madan-Swain.

\section{Supplemental Information}

\section{Previous Presentations}

Portions of this work were presented in poster form at the 46th Annual Meeting of the AANS/CNS Section on Pediatric Neurological Surgery, Houston, TX, November 28-December 1, 2017; and the 2018 AANS Annual Scientific Meeting, New Orleans, LA, April 28-May 2, 2018.

\section{Correspondence}

Brandon G. Rocque: University of Alabama at Birmingham, AL. brandon.rocque@childrensal.org. 